SIT-HEP/TM-30

\title{
Elliptic Inflation: Generating the curvature perturbation without slow-roll
}

\author{
Tomohiro Matsuda ${ }^{1}$ \\ Laboratory of Physics, Saitama Institute of Technology, \\ Fusaiji, Okabe-machi, Saitama 369-0293, Japan
}

\begin{abstract}
There are many inflationary models in which inflaton field does not satisfy the slow-roll condition. However, in such models, it is always difficult to generate the curvature perturbation during inflation. Thus, to generate the curvature perturbation, one must introduce another component to the theory. To cite a case, curvatons may generate dominant part of the curvature perturbation after inflation. However, we have a question whether it is unrealistic to consider the generation of the curvature perturbation during inflation without slow-roll. Assuming multi-field inflation, we encounter the generation of the curvature perturbation during inflation without slow-roll. The potential along equipotential surface is flat by definition and thus we do not have to worry about symmetry. We also discuss about KKLT models, in which corrections lifting the inflationary direction may not become a serious problem if there is a symmetry enhancement at the tip (not at the moving brane) of the inflationary throat.
\end{abstract}

\footnotetext{
${ }^{1}$ matsuda@sit.ac.jp
} 


\section{Introduction}

Among many benefits from the inflationary expansion that takes place in the early Universe, an important prediction of inflation would be the generation of a spectrum of the primordial perturbations. Such perturbations naturally arise from the zero point vacuum fluctuations in quantum fields, which are stretched during inflation to cover very large scales in our present Universe. In the standard scenario of the inflationary Universe, the observed density perturbation is produced by a light inflaton field that rolls slowly down its potential. At the end of inflation, the inflaton field oscillates about the minimum of its potential and decays to reheat the Universe. Adiabatic density perturbation is generated because the scale-invariant fluctuations of the light inflaton field are different in different patches. Generically, slow-roll models of inflation predict an almost scale-invariant and Gaussian distribution of primordial density perturbations.

We are not going back to historical developments, however it is easy to understand that the idea of hybrid inflation may provide us with a key idea to construct successful inflationary models [1]. In hybrid models, the end of inflationary expansion is a secondorder phase transition triggered by a trapped field (waterfall field). D-term inflation is an important application of this idea, which is found in the paradigm of supersymmetric particle cosmology. Furthermore, an important variant of D-term inflation is found in the paradigm of brane cosmology, which is called brane inflation [2, 3, 4]. From phenomenological viewpoints, brane models are sometimes categorized as models with large or intermediate extra dimensions (and vice versa) [5]. The idea of large extra dimensions is important for higher-dimensional models, because it may solve or weaken the hierarchy problem. In models with large extra dimensions, fields in the standard model(SM) are localized on a wall-like structure (maybe it is a brane), while the graviton propagates in the bulk. The discrepancy of the volume factor between gauge fields and gravitational fields explains the large hierarchy between gravity and gauge interactions.

Of course, it is an important challenge to find signatures of branes in cosmological observations. Historically, it has been discussed that studying the formation and the evolution of cosmological defects would provide us with important information about 
branes 6, 7]. ${ }^{2}$ Inflation models with low fundamental scale are discussed in ref. 9]. Scenarios of baryogenesis in such low-scale models are discussed in ref. [10, 11, 12, where defects play distinguishable roles. The curvatons [13, 14, 15] will play significant roles in these low-scale models 16, 17. Moreover, in ref. 17, it has been discussed that topological defects can play the role of the curvatons. Thus, defects in brane models such as monopoles, strings, domain walls and Q-balls are important [18, 19, 20, 21, 22, 23. It might be important to explain why defects other than strings can be produced in brane inflationary models, since (historically) it has been discussed by many authors that only strings are produced in brane inflationary models 24. It is not hard to understand that this conjecture is not so generic as it has been anticipated, as one can see from ref.[25]. Therefore, in order to find signatures of branes, it is important to consider other types of defects as well as cosmic strings. Our scenario of elliptic inflation is important, in a sense that it may provide us with another clue as to how one can find signature of branes from cosmological observations, as we will discuss in Sect.3.5.

When it appeared, D-term inflation was believed to circumvent the well-known etaproblem of supergravity models of inflation based on F-terms. Thus brane inflation, which is in a sense a variant of D-term inflation, was believed to circumvent the eta-problem. However, a similar problem arose later again in brane paradigm. The problem was that the very mechanism that lifts the moduli potential was found to lift inflaton candidates and violate slow-roll conditions 26. Directions that are protected by exact global symmetry may be expected to remain flat, however it is still very difficult to find actual symmetry that can survive after moduli stabilization and can be used to construct a successful inflationary scenario. ${ }^{3}$ From the perspective we discussed above, we believe that finding new mechanism for generating the curvature perturbation without slow-roll inflaton is quite important. In general, the superhorizon spectrum of perturbations is thought to be generated by the amplification of the quantum fluctuations of a light inflaton field, whose mass is much smaller than the Hubble constant during inflation. This is because the quantum fluctuations of the field can reach and exit the horizon only if its Compton wavelength is larger than the horizon during inflation. However, this happens only if

\footnotetext{
${ }^{2}$ Supergravity provides a natural mechanism for removing cosmological domain walls 8 .

${ }^{3}$ There are interesting approaches in ref. [27] and [28].
} 
the inflaton is effectively massless, i.e. only if $m_{I} \ll H_{I}$. The above condition seems to conflict with our aim in this paper. Luckily, we know alternatives for the slow-roll inflaton. The curvatons and their variants can generate the curvature perturbation after inflation 13, 14, even if there is no slow-roll during inflation. However, this mechanism still requires flat direction that is supposed to have non-trivial properties. Thus, we think it is still important to find models for generating the curvature perturbation without using neither slow-roll inflaton nor the curvatons. The situations that we will consider in this paper are both general and useful. Our mechanism can be utilized in many kinds of phenomenological models in which slow-roll condition is inevitably violated. As we are considering inflation without slow-roll, the e-foldings of our model may be short, which will require compensation by another period of inflationary expansion.

\section{Elliptic Inflation}

Consider two inflaton fields $\left(\phi_{1}, \phi_{2}\right)$ with a hybrid type of potential of the form ${ }^{4}$

$$
V\left(\phi_{1}, \phi_{2}, \sigma\right)=\frac{1}{2} m^{2}\left(\phi_{1}^{2}+A \phi_{2}^{2}\right)+\frac{\lambda_{1}}{2}\left(\phi_{1}^{2}+B \phi_{2}^{2}\right) \sigma^{2}+\frac{\lambda_{2}}{4}\left(\sigma^{2}-M^{2}\right)
$$

where $\phi_{i}(i=1,2)$ and $\sigma$ are taken to be real scalar fields. For later convenience, we introduce a field $\phi_{r}$

$$
\phi_{r}^{2} \equiv \phi_{1}^{2}+A \phi_{2}^{2}
$$

and mass

$$
m_{\sigma}^{2} \equiv \lambda_{2} M^{2}
$$

The above potential has global minima at

$$
(\phi,|\sigma|)=(0, M)
$$

and an unstable saddle point at

$$
(\phi, \sigma)=(0,0)
$$

\footnotetext{
${ }^{4}$ In this section we outline a comprehensive strategy for obtaining inflationary expansion without slowroll. One may be disappointed to see what everyone has already seen. However, this is not the point that we want to discuss in this paper. What we want to address in this paper is not the way how one can obtain inflationary expansion without slow-roll, but how one can obtain the curvature perturbation without neither slow-roll inflaton nor the curvatons. We will consider the latter issue in Sect.3.
} 
One may be disappointed to see the above potential, since it looks quite similar to the one that has been used in usual hybrid inflation. ${ }^{5}$ However, we never assume slow-roll condition for the inflation field, except for "built-in models" that we will consider in Sect.3.4

Since the effective mass squared of the waterfall field $\sigma$ is given by $m_{\sigma}^{2}(\phi)=$ $\lambda_{1}\left(\phi_{1}^{2}+B \phi_{2}^{2}\right)-m_{\sigma}^{2}$, the waterfall field $\sigma$ remains at $\sigma=0$ as far as the following condition is satisfied;

$$
\phi_{1}^{2}+B \phi_{2}^{2}>\phi_{c}^{2} \equiv m_{\sigma}^{2} / \lambda_{1}
$$

Suppose that initially the system lies in the above region (2.6) and $\sigma$ is at the quasi-stable point $\sigma=0$. In the case where the false vacuum density is dominated by a scalar potential

$$
V_{I}=\frac{\lambda_{2}}{4} M^{4}+\frac{1}{2} m^{2} \phi_{r}^{2}
$$

and if $\frac{\lambda_{2}}{4} M^{4}>\frac{1}{2} m^{2} \phi_{r}^{2}$, there would be a period of inflationary expansion even if there is no slow-roll inflaton field. During this period, according to the Friedman equation, we have $H_{I} \simeq V_{I}^{1 / 2} / M_{p}$. Since we are not expecting slow-roll inflation, the Hubble constant during inflation can be much smaller than the masses of the fields $\phi_{i}, i=1,2 .{ }^{6}$ During the period of inflation, the Klein-Gordon equation for $\phi_{i}$ is

$$
\ddot{\phi}_{i}+3 H \dot{\phi}_{i}+\frac{\partial V}{\partial \phi_{i}}=0
$$

where dots denote the derivatives with respect to the cosmic time $t$. The above equation has solution for the background dynamics that can be computed. The background field dynamics is given by

$$
\phi_{i}(t) \propto e^{\alpha_{i} t}
$$

where

$$
\alpha_{i}=\frac{3}{2} H_{I}\left[1 \pm\left\{1-\frac{4 m_{i}^{2}}{9 H_{I}^{2}}\right\}^{1 / 2}\right] .
$$

Here the masses of the fields are denoted by $m_{i}$.

\footnotetext{
${ }^{5}$ Hybrid inflation without slow-roll is already discussed in ref. 29 for single-field inflation.

${ }^{6}$ We consider two fields, $\phi_{1}$ and $\phi_{2}$. We know both fields may play the role of inflaton, however the actual e-foldings is almost determined by either field. Thus, we use the word "inflaton" for the field that determines the total e-foldings. In this paper, we use the field $\phi_{1}$ as the inflaton.
} 


\section{$2.1 \quad$ Fast-roll}

The idea of fast-roll inflation is first advocated by Linde in ref.[30]. Fast-roll inflation occurs during a period of inflation when the mass of the inflaton field is comparable to the Hubble parameter. In our case, as suggested by eq.(2.10), fast-roll occurs when

$$
m_{i} \leq \frac{3}{2} H_{I}
$$

There are two solutions to the Klein-Gordon equation (2.9), both of which are exponentially decreasing with time. Here we use the one with the negative sign, since the one with the opposite sign corresponds to the fast-decaying mode that rapidly disappears. Therefore, the solution that survives is

$$
\phi_{i}=\phi_{i 0} e^{-F_{i} N t}
$$

where $N(t)$ is the number of the e-folds elapsed during inflation, and $F_{i}$ is given by

$$
F_{i} \equiv \frac{3}{2}\left[1-\left\{1-\frac{4 m_{i}^{2}}{9 H_{I}^{2}}\right\}^{1 / 2}\right] .
$$

Assuming that (1) $\phi_{1}$ comes later than $\phi_{2}$, and (2) $\phi_{1}$ triggers waterfall stage when $\phi_{2}$ is already in the region $\left|\phi_{2}\right| \ll \phi_{c} / \sqrt{B}$, the total number of e-folds elapsed during fast-roll inflation is given by

$$
N_{F}=F_{1}^{-1} \ln \left(\frac{\phi_{1,0}}{\phi_{1 e}}\right)
$$

where $\phi_{1 e}^{2}=\phi_{c}^{2}-B \phi_{2 e} \simeq \phi_{c}^{2}$ denotes the end-point of fast-roll inflation. See also Fig \.

\subsection{Locked oscillation}

Locked inflation was advocated in ref.[31] using a potential of the form eq.(2.1). In the original model of locked inflation, an inflaton field oscillates on top of the false vacuum $\sigma=0$ during inflationary expansion. As is seen from (2.10), the boundary between fastroll inflation and locked inflation is at $m_{i}=\frac{3}{2} H_{I}$. In the case where $m_{i}>\frac{3}{2} H_{I}$, one obtains oscillating inflaton field that locks $\sigma$ at the origin. Then, the Klein-Gordon equation is solved by an equation

$$
\phi_{i}=\bar{\phi}_{i}(t) \cos \left(\beta_{i} t\right),
$$




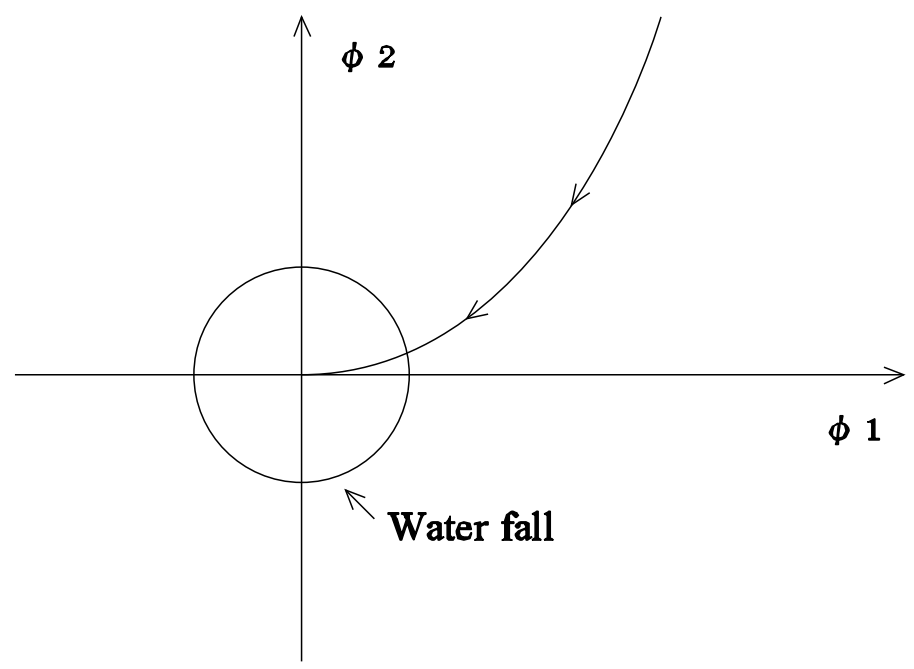

Figure 1: A trajectory $\left(\phi_{1}(t), \phi_{2}(t)\right)$ during inflationary expansion. We took a specific condition $m_{i}<\frac{3}{2} H_{I}$ (fast-roll) for $i=1,2$. In the case where $\phi_{2}$ becomes sufficiently small before the end of inflation, the total e-foldings are determined by the evolution of $\phi_{1}$. We know in such cases one tends to think that the secondary field $\phi_{2}$ plays no role in generating the curvature perturbation. However, this is not the case, as we will explain below.

where

$$
\beta_{i}=H_{I} \sqrt{\frac{m_{i}^{2}}{H_{I}^{2}}-\frac{9}{4}} .
$$

The amplitude of the oscillation decreases with time, which becomes

$$
\overline{\phi_{i}}(t)=\phi_{i, 0} e^{-\frac{3}{2} N(t)}
$$

The total number of e-foldings is given by

$$
N_{L}(t)=\frac{2}{3} \ln \left(\frac{\phi_{10}}{\phi_{1 e}}\right),
$$

where we neglected effects from parametric resonance, which may or may not be significant depending on the model we will consider.

It may be important to note that there is a significant discrepancy between the usual oscillation in locked models and the one considered in our model. In our case, oscillating field does not necessarily hit the core region (i.e. $\sqrt{\phi_{1}^{2}+B \phi_{2}^{2}}<\phi_{c}$ ) during its oscillation. We show in Fig 2 what happens if the oscillation occurs during fast-roll inflation. 


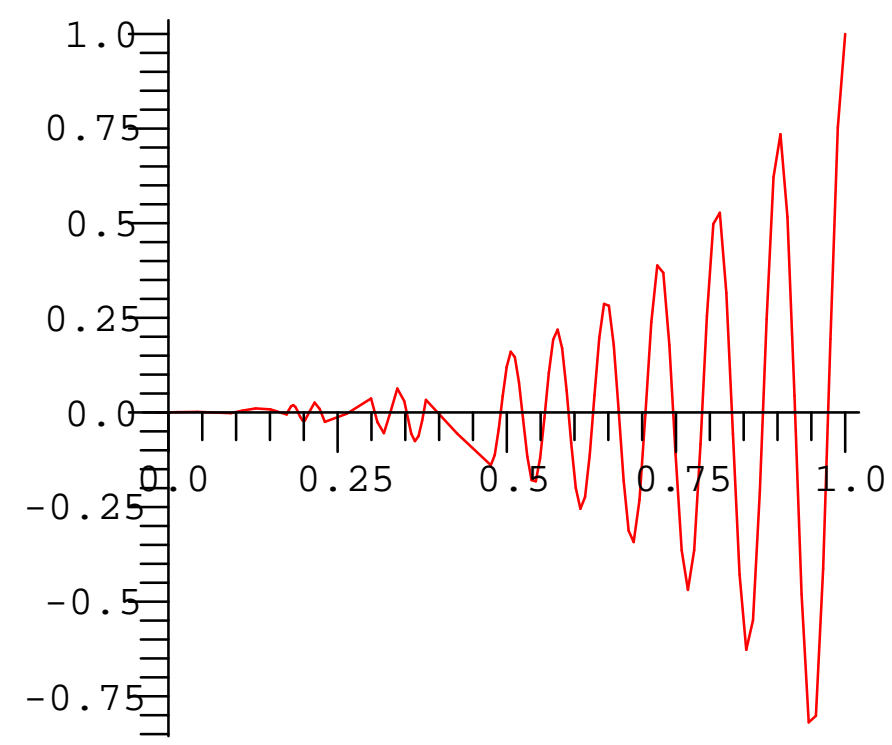

Figure 2: Artist's view of a trajectory $\left(\phi_{1}(t), \phi_{2}(t)\right)$ during inflationary expansion. We took a specific condition $m<\frac{3}{2} H_{I}$ (fast-roll) for $\phi_{1}$ and $\sqrt{A} m>\frac{3}{2} H_{I}$ (locked) for $\phi_{2}$. In this case, parametric resonance is suppressed even if the oscillation of the field $\phi_{2}$ is efficient at the beginning, since $\phi_{2}$ cannot hit the "core".

\section{$2.3 \quad$ Fast-roll after slow-roll}

One may consider a case where the vacuum energy (2.7) is dominated by a mass term of the field $\phi_{1}$. This may happen in the outer region where $\phi_{1}$ is large. See also Fig 3 .

Furthermore, if $\phi_{1}$ is as large as the Planck scale, there will be a stage of slow-roll inflation, as is always seen in conventional scenarios of chaotic inflation. In this case, fast-roll (or locked) inflation starts after the end of slow-roll inflation and helps the precedent inflation by adding extra e-foldings or reducing the vacuum energy and the reheat temperature.

\section{Models}

Assume that during inflationary period the potential is given by

$$
V\left(\phi_{1}, \phi_{2}\right)=\frac{1}{2} m_{1}^{2} \phi_{1}^{2}+\frac{1}{2} m_{2}^{2} \phi_{2}^{2}
$$




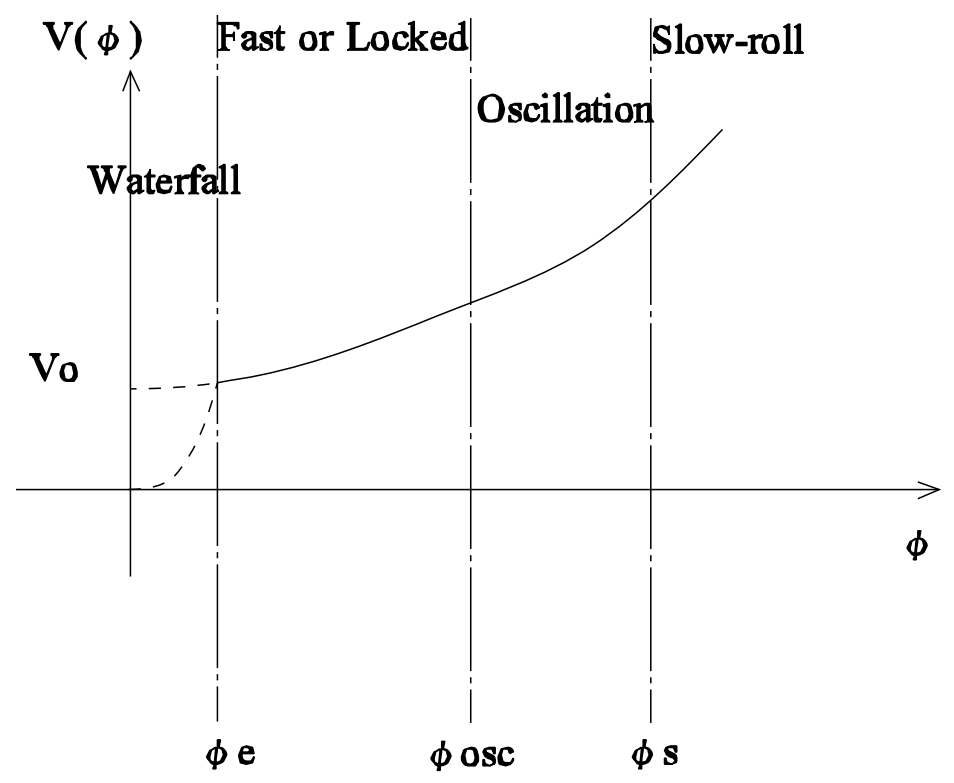

Figure 3: $\phi$ rolls slowly at a distance $\phi>\phi_{s} \sim M_{p}$, where mass term dominates the vacuum energy. Mass term still dominates the vacuum energy if $\phi$ is larger than $\phi_{\text {osc }}$, where $\phi_{o s c}$ is given by $\phi_{o s c} \equiv \sqrt{2 V_{0}} / m$. During the intermediate stage $\phi_{o s c}<\phi<\phi_{s}$ where mass term dominates the vacuum energy while slow-roll condition is violated, $\phi$ oscillates like curvatons. Then, fast-roll (or locked) inflation starts at $\phi=\phi_{o s c}$.

Introducing a complex field $\hat{\phi} \equiv \phi_{1}+i \hat{A} \phi_{2}$, where $\hat{A}$ is the ratio given by $\hat{A}=m_{2} / m_{1}$, we can rewrite the potential as

$$
V(\hat{\phi})=\frac{1}{2} m_{1}^{2}|\hat{\phi}|^{2} .
$$

Let me introduce a scalar field $\varphi$ that is given by

$$
\hat{\phi} \equiv v e^{i \frac{\varphi}{v}},
$$

where $v$ is defined as $v=|\hat{\phi}|$. Now the definition of the equipotential surface is

$$
V=\frac{1}{2} m_{1}^{2} v^{2}=\text { const. }
$$

and the field along the equipotential surface is $\varphi$. We can calculate the potential for the field $\varphi$ that is defined along the equipotential surface, which is given by

$$
V(\varphi)=\frac{1}{2} m_{1}^{2} v^{2}=\text { const } .
$$


It is easy to calculate the first derivative with respect to the field $\varphi$, which is given by

$$
\frac{\partial V(\varphi)}{\partial \varphi}=0
$$

and the second derivative is given by

$$
\frac{\partial^{2} V(\varphi)}{\partial \varphi^{2}}=0
$$

which shows that along these direction perturbations of the order of $H / 2 \pi$ will be generated, since the second derivative is vanishing along the equipotential surface.

In this paper, we will consider cases in which $\phi_{1}$ is an actual inflaton that determines e-foldings, while $\phi_{2}$ is an additional field that is not relevant to the e-foldings. ${ }^{7}$

If the fluctuations along the equipotential surface are sufficiently small compared with $\phi_{i}$, one can decompose it along its tangent line. Then, the fluctuations will be given by

$$
\left(\delta \phi_{1}, \delta \phi_{2}\right) \simeq \frac{H_{I}}{2 \pi \phi_{r, 0}}\left(A \times \phi_{2,0}, \phi_{1,0}\right)
$$

where $\phi_{i, 0}$ denotes the value of $\phi_{i}$ at the time when fluctuations exit horizon. In any case, the evolution of the fields $\phi_{i}$ is determined by Klein-Gordon equation (2.8). Fluctuation of a light field is defined on any spacetime slicing and is almost massless. After horizon exit, the vacuum fluctuations have generated classical perturbations, which vary slowly on the Hubble timescale and are expected to be Gaussian. These classical perturbations have the spectrum $\mathcal{P}_{\delta \phi}^{1 / 2}=H_{k} / 2 \pi$, where $H_{k}$ denotes the Hubble constant $H$ at horizon exit. The evolution of $\zeta$ before horizon entry is described by the famous $\delta N$ formalism [32, 33, 34]. The time-dependent curvature perturbation smoothed on the scale $k$ is

$$
\zeta(x, t)=\delta N\left(k, \phi_{i}(x), \rho(t)\right)
$$

where $N$ denotes the number of e-foldings from the epoch of horizon exit to an epoch when the energy density has a value $\rho .{ }^{8}$ Using the above idea of the $\delta N$ formalism, Lyth

\footnotetext{
${ }^{7}$ To be more precise, $\left|\phi_{2}\right| \ll \phi_{c} / \sqrt{B}$ occurs before the end of inflation.

${ }^{8}$ Note that in general $N$ depends on both $\phi_{i}$ and $\dot{\phi}_{i}$, but if $t$ is during slow-roll inflation one can use the slow roll approximation to eliminate the dependence on $\dot{\phi}_{i}$. In our case, however, we cannot simply ignore the dependence because we are not thinking about slow-roll inflation. In the following calculations we will omit the $\dot{\phi}_{i}$-dependence if it is obvious. Be sure that we are not omitting the $\dot{\phi}_{i}$-dependence because of the slow-roll conditions, but considering the explicit form of $N$ to see the $\dot{\phi}_{i}$-dependence.
} 
made a concrete example for generating curvature perturbation at the end of inflation 33 . To illustrate the idea, let us consider a model in which there is a unique inflationary trajectory. Then, $\phi_{i}$ becomes a unique function of the proper time $\tau$ up to a shift in the origin. As a result, all functions of $\phi_{i}$ will also be unique, including the energy density

$\rho=V+\frac{d^{2} \phi_{i}}{d^{2} \tau}$. In this case, at each position of the Universe inflation ends at $\phi_{I}=\phi_{I e}$ that is a constant and is independent of position. The novel possibility discussed in ref. [33] is that $\phi_{I e}$ depends on some other field $\phi_{a d d}$ whose potential is practically flat. Then, $\phi_{e}$ depends on spatial position because of the perturbation $\delta \phi(x)_{a d d}$. This changes the e-foldings from a spacetime slice of uniform density just before the end of inflation to a spacetime slice of uniform density just after the end of inflation. In this paper, the above change of the e-folds is denoted specifically by $\delta N_{\Delta} \equiv \zeta_{\Delta}(x)$. This quantity $\zeta_{\Delta}$ is the contribution to the curvature perturbation generated at the end of inflation.

We must be careful about the evolution of fluctuations after horizon exit. Although $\delta N_{\Delta}$ is produced at the end of inflation, fluctuations $\delta \phi_{a d d}$ are produced much before the end of inflation and have exited horizon during inflation. Here we would make a modest assumption that the value $\delta \phi_{e}(t) / \phi_{e}(t)$ is almost a constant during the era [1, 15]. Thus, the fluctuation of $\phi_{a d d}$ at the end of inflation is given by

$$
\delta \phi_{a d d}\left(t_{e}\right) \simeq \frac{H_{I}\left(t_{k}\right)}{2 \pi}\left(\frac{\phi_{a d d}\left(t_{e}\right)}{\phi_{a d d}\left(t_{k}\right)}\right),
$$

where $t_{k}$ and $t_{e}$ are the time when fluctuation exits horizon and the time when inflation ends, respectively. If the field $\phi_{a d d}$ is much lighter than $\phi_{I}, \delta \phi_{a d d}\left(t_{e}\right) \simeq \delta \phi_{a d d}\left(t_{k}\right)$ is a conceivable approximation.

\subsection{Fast $\times$ flat}

The easiest example would be that $\phi_{1}$ is fast-roll inflaton, while $\phi_{2}$ is flat direction that does not roll during inflation. To be more precise, $\phi_{1}$ and $\phi_{2}$ corresponds to $\phi_{I}$ and $\phi_{a d d}$ in the above argument, respectively. In our scenario of elliptic inflation, this situation corresponds to $A \ll 1$ and $\phi_{2 e} \ll \phi_{c} / \sqrt{B}$, where the equipotential surface becomes a squeezed ellipsoid. Initially, the equipotential surface is given by

$$
\phi_{0}^{2} \equiv \phi_{1,0}^{2}+A \phi_{2,0}^{2}
$$


As one can see from Fig 4 , the fluctuation along equipotential surface is almost $\delta \phi_{2} \simeq$ $H_{I} / 2 \pi$ due to the above conditions $A \ll 1$ and $\phi_{2 e} \ll \phi_{c} / \sqrt{B}$.

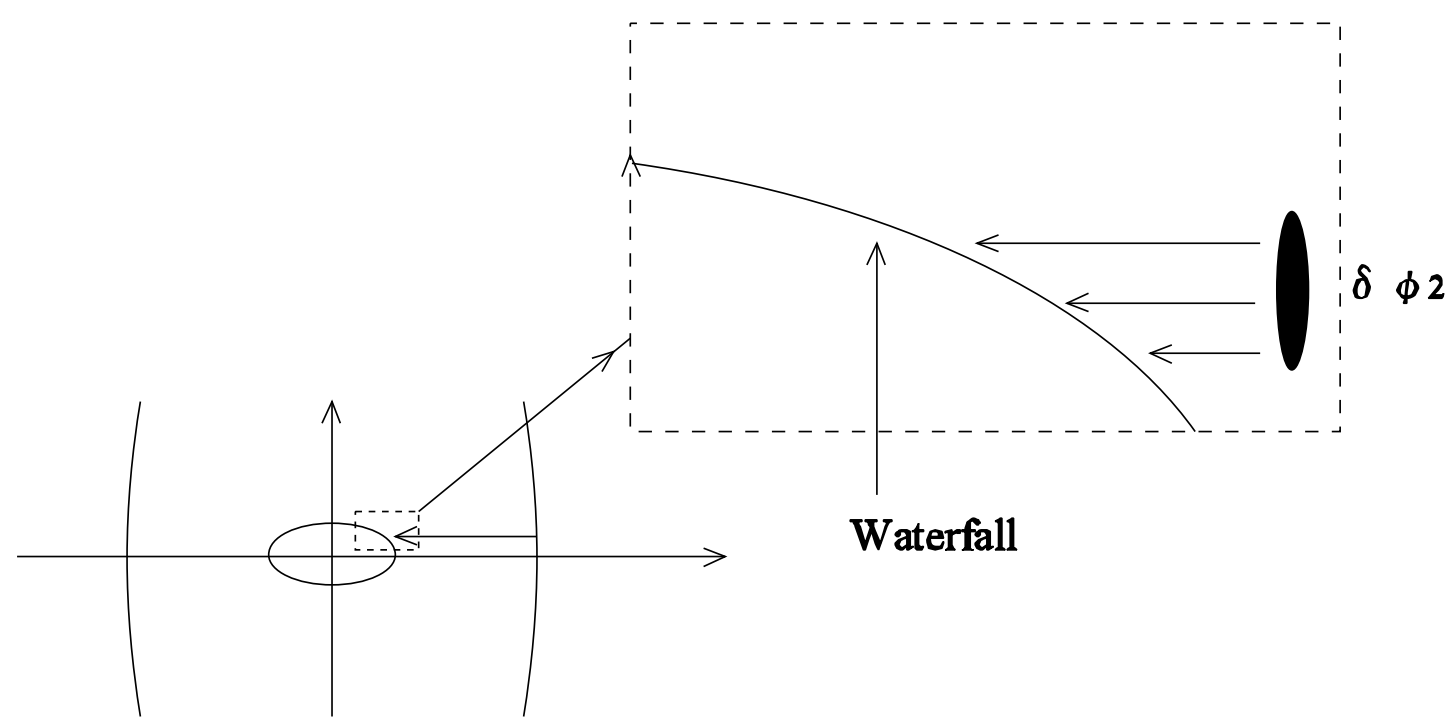

Figure 4: $\delta \phi_{2}$ leads to the delay of the waterfall stage. This phenomenon looks similar to the usual back-and-force perturbation of the single-field model, although inflaton field $\phi_{1}$ does not roll slowly. Of course, unlike the usual single-inflaton models, there is no reason that one can naively believe in (almost) scale-invariant and (almost) Gaussian perturbations. It is lucky that we could find scale-invariant Gaussian perturbation in a certain parameter region of this model.

Because of the assumption $A \ll 1$ for the potential (2.1), there is a large hierarchy between $m_{1}$ and $m_{2}$. Thus, we can neglect the roll of the secondary field $\phi_{2}$ during inflation. Then, we can calculate $\zeta_{\Delta}(x)$ using the method advocated in ref. 33, which takes the form of

$$
\zeta_{\Delta}(x)=\frac{\partial N_{\Delta}}{\partial \phi_{1}} \frac{\partial \phi_{1}}{\partial \phi_{2}} \delta \phi_{2}+\frac{1}{2}\left\{2 \frac{\partial^{2} N_{\Delta}}{\partial \phi_{1}^{2}}\left(\frac{\partial \phi_{1}}{\partial \phi_{2}}\right)^{2}+\frac{\partial N_{\Delta}}{\partial \phi_{1}} \frac{\partial^{2} \phi_{1}}{\partial \phi_{2}^{2}}\right\}\left(\delta \phi_{2}\right)^{2} .
$$

We do not assume slow-roll for the inflaton field $\phi_{1}$. It is amazing that this mechanism for generating the curvature perturbation still works to generate scale-invariant and Gaussian perturbation, even in the case where $\phi_{1}$ is not a slow-roll inflaton field. ${ }^{9}$ As we will show later in this paper, this model presents a peculiar limit of our more generalized scenario.

\footnotetext{
${ }^{9}$ This possibility is already suggested in ref. 33 .
} 
If $\phi_{1}$ is a fast-roll inflaton field, it is easy to obtain

$$
\begin{aligned}
\left.\frac{\partial N_{\Delta}}{\partial \phi_{1}}\right|_{e} & =-\frac{1}{F_{1} \phi_{1 e}} \\
\left.\frac{\partial^{2} N_{\Delta}}{\partial \phi_{1}^{2}}\right|_{e} & =\frac{1}{F_{1} \phi_{1 e}^{2}},
\end{aligned}
$$

and

$$
\begin{aligned}
\left.\frac{\partial \phi_{1}}{\partial \phi_{2}}\right|_{e} & =-B \frac{\phi_{2 e}}{\phi_{1 e}} \\
\left.\frac{\partial^{2} \phi_{1}}{\partial \phi_{2}^{2}}\right|_{e} & =-\frac{B}{\phi_{1 e}}\left(\frac{\phi_{2 e}^{2}}{\phi_{1 e}^{2}}+1\right) .
\end{aligned}
$$

As a result, $\zeta_{\Delta}(x)$ is given by

$$
\zeta_{\Delta}(x)=\frac{B H_{I}}{2 \pi F_{1} \phi_{1 e}^{2}}\left[\phi_{2 e}+\frac{H_{I}}{4 \pi}\left(\frac{2 B \phi_{2 e}^{2}}{\phi_{1 e}^{2}}+\frac{\phi_{2 e}^{2}}{\phi_{1 e}^{2}}+1\right)\right],
$$

where we used $\phi_{2,0} \simeq \phi_{2 e}$ to obtain $\delta \phi_{2 e}$. From the above equation, one can easily understand that non-Gaussianity parameter becomes large if $\phi_{2 e} \ll H_{I}$. As is seen from the above result (3.15), the curvature perturbation does not depend on the value of $\phi_{i 0}$. Thus, at least in the limiting case where $A \ll 1$ is assumed, $\zeta_{\Delta}(x)$ can become scaleinvariant in a sense that it does not depend on the time when fluctuations exit horizon. Application of the above scenario is discussed in Sect.3.5 as a solution to the eta-problem in brane inflationary models. On the other hand, if $A$ is not so small, $\zeta_{\Delta}(x)$ may depend on $\phi_{i 0}$, as we will discuss below.

\section{$3.2 \quad$ Fast $\times$ fast}

What happens if we remove the above condition $A \ll 1$ ? Assume that the fields are initially at $\left(\phi_{1,0}, \phi_{2,0}\right)$ and also that the order of magnitude of fluctuations are much smaller than their initial values. Then, one can decompose fluctuations along the tangent line of the equipotential surface. The fluctuations along the tangent line would be given by eq.(3.8). Although both $\phi_{i}(t)$ and $\delta \phi_{i}(t)$ will evolve during inflation, it is conceivable to assume that the ratio $\delta \phi_{i} / \phi_{i}$ is a constant during the inflationary period [15]. Following the $\delta N$ formalism 33 , it is easy to calculate

$$
\begin{aligned}
\delta N= & \frac{\partial N_{\Delta}}{\partial \phi_{1}} \delta \phi_{1}+\frac{\partial N_{\Delta}}{\partial \phi_{1}} \frac{\partial \phi_{1}}{\partial \phi_{2}} \delta \phi_{2} \\
& +\frac{1}{2} \frac{\partial^{2} N_{\Delta}}{\partial \phi_{1}^{2}}\left(\delta \phi_{1}\right)^{2}+\frac{1}{2}\left\{2 \frac{\partial^{2} N_{\Delta}}{\partial \phi_{1}^{2}}\left(\frac{\partial \phi_{1}}{\partial \phi_{2}}\right)^{2}+\frac{\partial N_{\Delta}}{\partial \phi_{1}} \frac{\partial^{2} \phi_{1}}{\partial \phi_{2}^{2}}\right\}\left(\delta \phi_{2}\right)^{2} .
\end{aligned}
$$


It would be important to note here that the initial fluctuations $\delta \phi_{i}$ are not vanishing even if their masses are heavy. See Fig 5 for a perspective sketch of what happens in our scenario. Amazingly, our mechanism for generating the curvature perturbation works

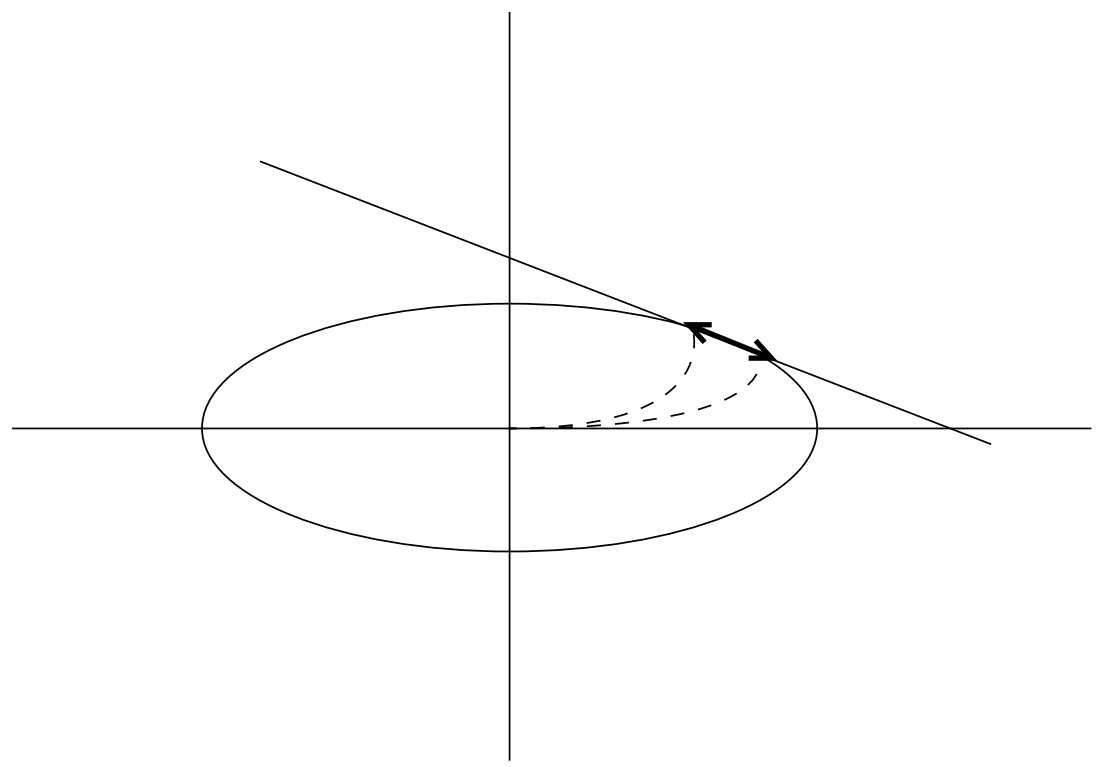

Figure 5: Fluctuations can appear along the equipotential surface even if the potential is steep for both $\phi_{1}$ and $\phi_{2}$.

even if both fields are massive. We can use previous results

$$
\begin{aligned}
\left.\frac{\partial N_{\Delta}}{\partial \phi_{1}}\right|_{e} & =-\frac{1}{F_{1} \phi_{1 e}} \\
\left.\frac{\partial^{2} N_{\Delta}}{\partial \phi_{1}^{2}}\right|_{e} & =\frac{1}{F_{1} \phi_{1 e}^{2}},
\end{aligned}
$$

and

$$
\begin{aligned}
\left.\frac{\partial \phi_{1}}{\partial \phi_{2}}\right|_{e} & =-B \frac{\phi_{2 e}}{\phi_{1 e}} \\
\left.\frac{\partial^{2} \phi_{1}}{\partial \phi_{2}^{2}}\right|_{e} & =-\frac{B}{\phi_{1 e}}\left(\frac{\phi_{2 e}^{2}}{\phi_{1 e}^{2}}+1\right)
\end{aligned}
$$

to calculate the curvature perturbation. It gives

$$
\begin{aligned}
\zeta_{\Delta}(x)= & -\frac{1}{F_{1} \phi_{1 e}}\left(\frac{H_{I} u_{1}}{2 \pi} \frac{\phi_{1 e}}{\phi_{1,0}}\right)+\frac{1}{F_{1} \phi_{1 e}}\left(B \frac{\phi_{2 e}}{\phi_{1 e}}\right)\left(\frac{H_{I} u_{2}}{2 \pi} \frac{\phi_{2 e}}{\phi_{2,0}}\right) \\
& \frac{1}{2 F_{1} \phi_{1 e}^{2}}\left(\frac{H_{I} u_{1}}{2 \pi} \frac{\phi_{1 e}}{\phi_{1,0}}\right)^{2} \\
& \frac{1}{F_{1} \phi_{1 e}^{2}}\left(\frac{B \phi_{2 e}}{\phi_{1 e}}\right)^{2}\left(\frac{H_{I} u_{2}}{2 \pi} \frac{\phi_{2 e}}{\phi_{2,0}}\right)^{2}-\frac{B}{2 F_{1} \phi_{1 e}^{2}}\left(\frac{\phi_{2 e}^{2}}{\phi_{1 e}^{2}}+1\right)\left(\frac{H_{I} u_{2}}{2 \pi} \frac{\phi_{2 e}}{\phi_{2,0}}\right)^{2}
\end{aligned}
$$


where we have defined $u_{1} \equiv \frac{A \times \phi_{2,0}}{\phi_{r 0}}$ and $u_{2} \equiv \frac{\phi_{1,0}}{\phi_{r 0}}$. The first line of eq.(3.19) vanishes when $A=B=1$, as is expected, while the second-order terms do not. Remember that we have truncated higher order terms when we obtained the perturbation eq.(3.8). Now it is obvious that we should have included higher terms in eq.(3.8) to obtain exact result for second order terms in eq.(3.19). On the other hand, the result (3.15) is a good approximation, since we have assumed $A \ll 1$ in Sect.3.1. At this time, we are not interested in the second order terms in the above result. Obviously, the result (3.19) depends on the time when fluctuations exit horizon, since in the above equation there are terms that depend on $\phi_{i 0}$. Note that $\phi_{i 0}$ parameterizes the time when fluctuations exit horizon. According to eq.(3.19), perturbations produced at later stages of elliptic inflation have much larger amplitudes in its spectrum, thus it has a blue spectrum. This is a problem for the people who want to generate the curvature perturbation only from elliptic inflation, while it seems a good idea to obtain a tilted spectrum, at least for the people who want to embed this idea into a model where the scale-invariant curvature perturbation is generated by another mechanism. For example, one may consider a model where slow-roll inflation occurs before elliptic inflation. We will discuss about this issue again in Sect.3.4. Of course, it may be a good idea to obtain a tilted spectrum embedding this model into a scenario with the curvatons [29]. In this case, one may assume that the large-scale perturbation is generated by the curvatons, while the perturbation generated by elliptic inflation becomes large in its short-scale side, thus generating a tilted spectrum.

\section{$3.3 \quad$ Fast $\times$ locked}

It would be useful to consider the opposite limit of $A \ll 1$, where the additional field $\phi_{2}$ is very massive. Of course, we do know it is quite normal to think that an additional field cannot play any role in generating the curvature perturbation if it is heavier than the inflaton field. However, this anticipation is not always true in our scenario. The idea we will consider here is schematically sketched in Fig6, where $B \ll 1$ and $A \gg 1$ is assumed. In this case, we can take $u_{2} \simeq 0$ and $u_{1} \simeq 1$, and can neglect all terms that are proportional to $u_{2}$. Then, from eq.(3.19) we obtain

$$
\zeta_{\Delta}(x) \simeq \frac{H_{I}}{2 \pi F_{1} \phi_{1,0}}-\frac{\left(H_{I}\right)^{2}}{8 \pi^{2} F_{1} \phi_{1,0}^{2}},
$$




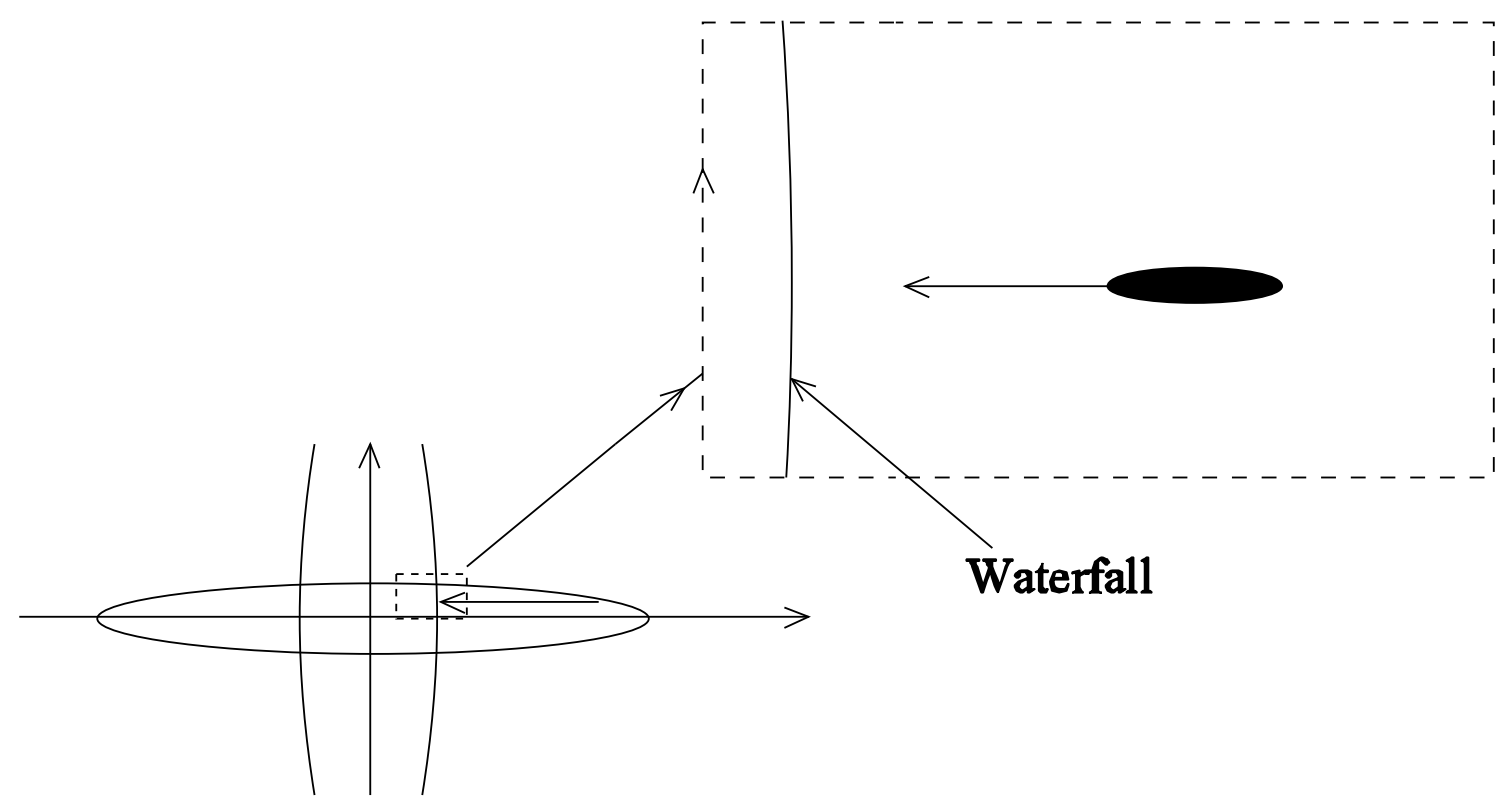

Figure 6: The component of the fluctuation is almost $\delta \phi_{1} \simeq \frac{H_{I}}{2 \pi}$ because we took a limit $A \gg 1$ (i.e. the additional field $\phi_{2}$ is very heavy.). The fluctuation $\delta \phi_{1}$ leads to the delay of the waterfall stage, which looks precisely the same as the usual back-andforce perturbation of slow-roll inflation. In this case, however, one cannot expect scale invariance because $\phi_{1}$ does not roll slowly.

where the higher terms that are hidden in eq.(3.8) can be neglected, since we are considering an extreme case with $A \gg 1$. This result is quite interesting. We have obtained a blue spectrum in an inflationary scenario without slow-roll, by just adding a heavy field $\phi_{2}$ that couples very weakly to $\sigma$. A plausible situation is that the secondary field $\phi_{2}$ oscillates rapidly during inflation, while $\phi_{1}$ play the role of the conventional fast-roll inflaton field. ${ }^{10}$ Then, fluctuations will be generated along the direction that is almost perpendicular to the oscillation. This direction is almost massless, like fluctuation of a falling raindrop. At the end of inflationary expansion, $\delta N_{\Delta}$ is produced by the fluctuation. Although the expectation value of the heavy field $\phi_{2}$ may already be vanishing at the end of inflation and may not appear explicitly in the effective action at this time, one cannot disregard the fluctuations induced by this field, which are generated and exited horizon during earlier period of inflation.

In some cases, one may consider fast-roll (or locked) inflation as a source for e-folds,

\footnotetext{
${ }^{10}$ See also Fig 2
} 
while expecting that the curvature perturbation is produced by the curvatons. Then, one will disregard the curvature perturbation that may be generated during the period of fast-roll (or locked) inflation. This scenario seems plausible, however the situation is not so simple as it has been anticipated before. To illustrate our idea, let us include a heavy field $\phi_{2}$ in addition to the fast-roll (or locked) inflaton field $\phi_{1}$. As we have seen above, a heavy field may oscillate at the beginning of inflation, producing a blue spectrum. In the large-scale side, the spectrum may be dominated by the curvature perturbation generated by the curvatons as expected, however its short-scale side may be dominated by the curvature perturbation generated at the end of fast-roll inflation. This scenario gives a tilted spectrum.

\subsection{Built-in scenarios}

As we have discussed above, it is not easy to obtain scale-invariant spectrum only from elliptic inflation. However, it would be interesting if elliptic inflation starts after a short period of slow-roll inflation. To illustrate our idea, let us assume that the (scale-invariant) perturbation is produced during a precedent stage of slow-roll inflation that generates minimum amount of e-foldings. Then, one obtains a tilted spectrum, which is due to the succeeding stage of elliptic inflation.

We think it is quite natural to expect the occurrence of fast-roll (or locked) inflation after the period of conventional slow-roll inflation. ${ }^{11}$ If so, the spectrum should have unusual tilt in its short-scale side. Today, the smallest scale accessible to large-scale structure observations is about $1 \mathrm{Mpc}$. This scale is equal to the Hubble radius about nine e-foldings after our present Hubble scale $a_{0} H_{0}$ exit horizon. Therefore, we need at least nine e-foldings of slow-roll expansion that generates scale-invariant spectrum, if it is to explain the observed large-scale structure.

Then, it would be interesting if one can find signatures of the succeeding stage of (secondary) inflation from the intermediate-scale cosmological observations. To examine

\footnotetext{
${ }^{11}$ For example, brane inflation is supposed to end when the inflaton mass exceeds the Hubble constant, which may be accompanied by fast (or locked) inflationary phase.
} 
this idea, let us consider a potential

$$
V_{I} \simeq V_{0}+\frac{1}{2} m \phi_{r}^{2}
$$

In the above calculations, we simply assumed that $V_{0}$ is much larger than mass terms $\frac{1}{2} m_{i}^{2} \phi_{i}^{2}$ and dominates the vacuum density during inflation. However, the above assumption is violated if $m$ and $\phi_{r}$ are large, and if the second term dominates the potential. In the case where the mass term dominates the vacuum density, the Hubble constant is given by $H_{I} \simeq m \phi_{r} / M_{p}$. This happens in the region where $\phi_{r} \geq \phi_{o o} \equiv \sqrt{V_{0}} / m$. We define $\phi_{s o}$ and $\phi_{s s}$ so that $H_{I}>m_{1}$ and $H_{I}>m_{2}$ are satisfied when $\phi_{r}>\phi_{s o} \simeq M_{p}$ and $\phi_{r}>\phi_{s s} \simeq \sqrt{A} M_{p}$, respectively. Elliptic inflation starts after slow-roll inflation, at the time when $\phi_{r}<\phi_{o o}$. $\phi_{1}$ (and not $\phi_{2}$ ) becomes slow-roll if $\phi_{s o} \leq \phi_{r} \leq \phi_{s s}$, and both $\phi_{1}$ and $\phi_{2}$ rolls slowly at a distance $\phi_{r}>\phi_{s s}$. See also Fig[

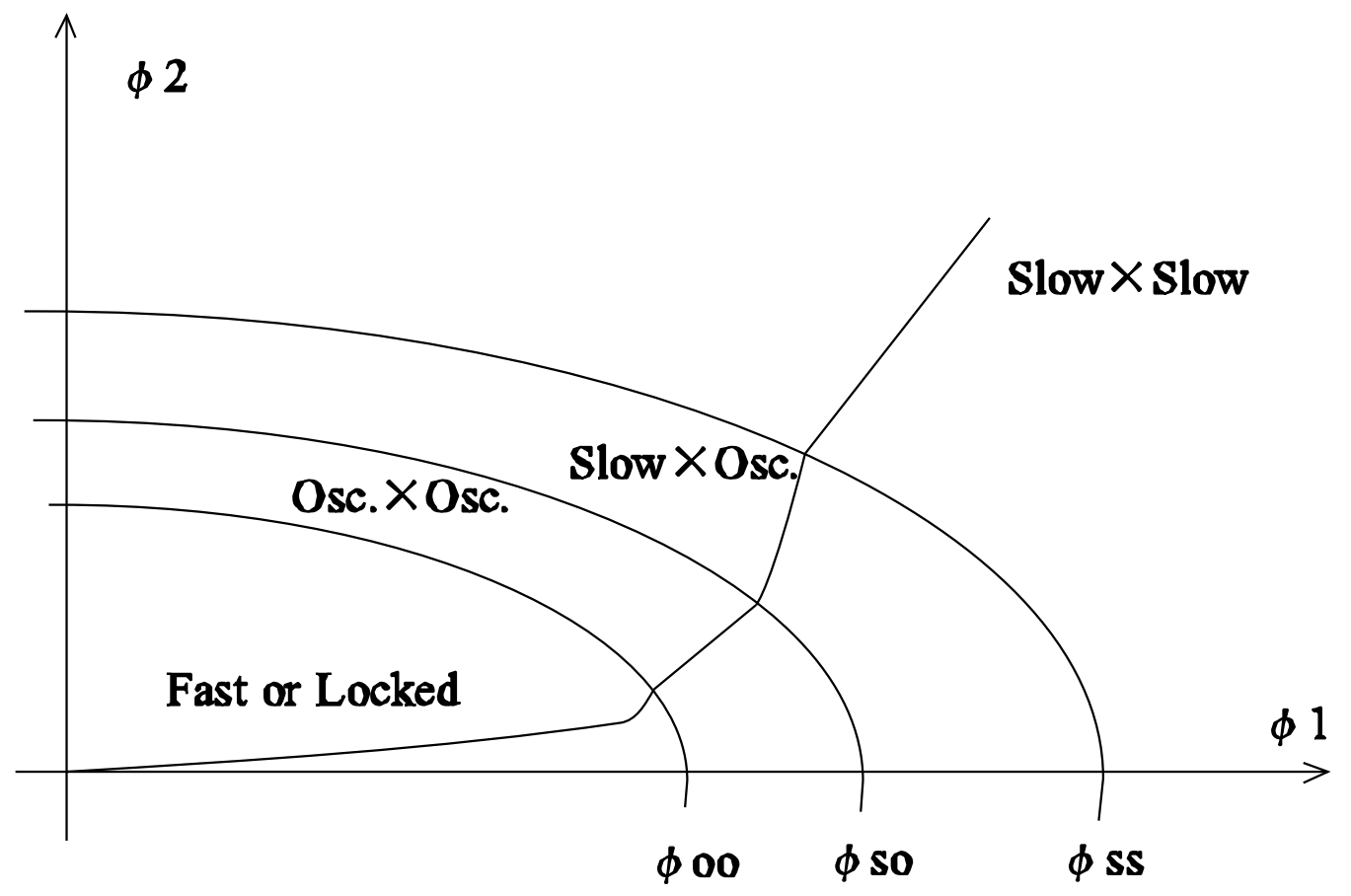

Figure 7: A schematic picture of the "built-in" scenario. Here we set $A>1$. Slow-roll phase at a distance is important in producing the large-scale spectrum, if there is no curvatons. 


\subsection{Brane inflation}

Although brane inflation is in a sense a variant of hybrid inflation, it may look different from the original. For example, in brane inflationary scenarios waterfall is triggered when the branes come closer than a critical distance. The mass of the waterfall field and the critical distance is determined by string dynamics on the branes, while the mass of inflaton (i.e. the mass of the position moduli) is determined by the global and local structure of the extra dimensions. Although brane inflation is a promising idea that explains how inflation is realized in brane cosmology, it is known that obtaining successful flat direction is quite difficult [26]. From the above viewpoints, it is important to examine if the curvature perturbation can be produced without slow-roll, at the same time without curvatons. It would be useful to note here that the "brane distance" is given by $r^{2}=\sum_{i} x_{i}^{2}$, where $x_{i}$ are the brane distances in the directions perpendicular to the inflating branes. In common case, there is no exact symmetry that protects all the masses of the position moduli. Thus, the equipotential surface of such potential is elliptic. As we have discussed above, one tends to think that the secondary field (or direction in extra dimensions perpendicular to the inflaton) plays no role in generating the curvature perturbation, if it is heavier than the primary inflaton field. However, this is not the case. One cannot disregard other inflaton candidates that are much heavier than the actual inflaton. Although brane inflation is supposed to end when the effective mass becomes as large as the Hubble constant, there may exist another period of inflationary expansion (fast or locked inflation) just after slow-roll inflation. In this sense, what we have discussed above in this paper provides us with a new inflationary scenario that makes it possible to generate a tilted spectrum in brane inflationary models. All flat directions can be lifted at the time of elliptic inflation. It does not disturb our mechanism for generating the curvature perturbation.

On the other hand, our mechanism is useful in solving the serious eta-problem in brane inflationary models. To illustrate our idea, let us consider a concrete example.

\subsubsection{Fast $\times$ flat inflation in a brane inflationary model}

The setting for our analysis is the dS background of KKLT[35. The stabilization of the Kähler modulus leads to a vacuum with a negative cosmological constant, however 
the vacuum can be lifted by adding an $\overline{D 3}$-brane that sits at the tip of the throat. An important consequence of the introduction of an $\overline{D 3}$-brane to the warped background is the appearance of new moduli that correspond to the position of the $\overline{D 3}$-brane on the compact space. The potential for these moduli in the KKLT background is rather curious. One can see from ref. [26, 35] that the $\overline{D 3}$-brane is not free to move in the throat direction since they have a potential proportional to the warp factor. This potential stabilizes the $\overline{D 3}$-brane at the tip of the throat. However, at the tip of the throat, the $\overline{D 3}$-brane can still move on the $S^{3}$. In the exact KS solution, $S^{3}$ is exact at the tip of the throat and the symmetry of $S^{3}$ is broken by placing the $\overline{D 3}$-brane as $S O(4) \rightarrow S O(3)$, which gives rise to three massless moduli. The moduli correspond to the three coordinates of the position of the $\bar{D} 3$-brane on the $S^{3}$. In generic background, there would be corrections from the UV-boundary, the Calabi-Yau manifold, since the background deviates from the original KS solution away from the tip. These corrections may explicitly break the $S O(4)$ symmetry, thus generate masses for the moduli. In ref. [36], one can find calculations for the masses that arise from the UV-corrections. Although the deformation of the theory at the UV-side generates massses for the moduli, the highest contribution is exponentially smaller than the typical mass scale at the tip. Therefore, in a brane inflationary scenario where the $\overline{D 3}$-brane is fixed at the tip of the throat while the moving brane comes from the root, the fluctuation of the $\overline{D 3}$-brane in the direction of $S^{3}$ becomes important. In our "Fast $\times$ flat scenario", the throat direction and the $S^{3}$ moduli correspond to the fields $\phi_{1}$ and $\phi_{2}$, respectively. ${ }^{12}$ Thus, the fluctuation of $\bar{D} 3$-brane at the tip can generate the curvature perturbation even if all the scalar fields parameterizing the position of the moving brane is lifted to have large masses. Although it will be rather difficult to explain total e-foldings only from single-shot inflation, one may add secondary inflation that may take place after fast-roll inflation. Thus, we can solve the eta-problem without using the curvatons. We think our idea is important in constructing inflationary models in KKLT and other scenarios.

\footnotetext{
${ }^{12}$ See also Fig 8
} 


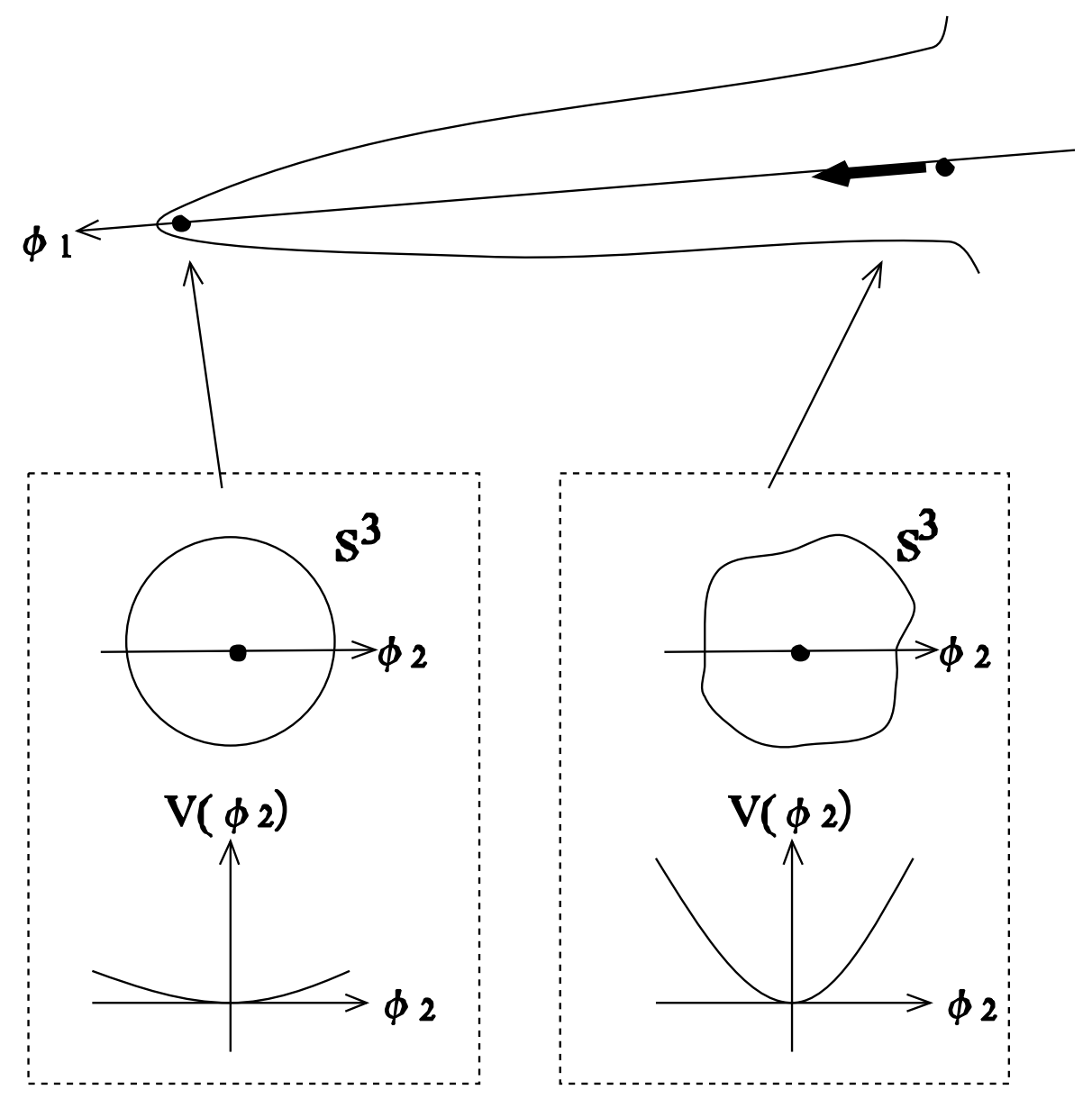

Figure 8: The deformation of the KS throat at the UV may explicitly breaks the $S O(4)$ symmetry, generating masses for the corresponding moduli. On the other hand, the deformation is milder at the tip of the throat, keeping the $S O(4)$ symmetry at the tip. Be sure that there is no reason that one must use the fluctuation of the moving brane in calculating the generation of the curvature perturbation.

\section{Conclusions}

The most important point is that an equipotential surface appears whenever there is additional inflaton that couples to waterfall field. Fluctuation appears along the equipotential surface and exits horizon during inflation. The equipotential surface is an ellipsoid, along which fields can fluctuate despite the large mass of the fields. Then, it induces fluctuation of the total number of e-folds at the end of inflation. The end line of hybrid inflation may also be ellipsoid, which becomes another source for the curvature perturbations. The spectrum of the curvature perturbation is determined by the masses and the couplings of 
the fields.

For brane inflationary models, our mechanism provides us with a new inflationary scenario that circumvent the eta-problem. It is possible to generate the curvature perturbation even in a model where all flat directions are lifted at the UV side of the KS throat. It is also notable that our mechanism can be used to obtain a tilted spectrum. The spectrum is determined by the masses of the position moduli that parameterize the position of the inflationary branes. The mechanism may provide us with an important clue as to how one can obtain information about the structure of extra dimensions from cosmological observations.

\section{Acknowledgment}

We wish to thank K.Shima for encouragement, and our colleagues in Tokyo University for their kind hospitality.

\section{References}

[1] A.R. Liddle and D.H. Lyth, Cosmological inflation and large scale structure, Cambridge, UK: Univ. Pr. (2000).

[2] G. R. Dvali and S. H. Henry Tye Brane inflation, Phys.Lett.B450(1999)72 hep-ph/9812483.

[3] C. Herdeiro, S. Hirano and R. Kallosh, String theory and hybrid inflation / acceleration, JHEP0112(2001)027 hep-th/0110271; K. Dasgupta, C. Herdeiro, S. Hirano and R. Kallosh, D3 / D7 Inflationary model and M theory, Phys.Rev.D65(2002)126002 hep-th/0203019, J. Garcia-Bellido, R. Rabadan and F. Zamora, Inflationary scenarios from branes at angles, JHEP 0201(2002)036 hep-th/0112147.

[4] T. Matsuda, Nontachyonic brane inflation, Phys.Rev.D67(2003)083519 hep-ph/0302035; T. Matsuda, F term, D term and hybrid brane inflation, JCAP 0311(2003)003 hep-ph/0302078; T. Matsuda, Thermal hybrid inflation in brane world, Phys.Rev.D68(2003)047702 hep-ph/0302253; T. Matsuda, Topological 
hybrid inflation in brane world, JCAP 0306(2003)007 hep-ph/0302204; T. Matsuda, Q ball inflation, Phys.Rev.D68(2003)127302 hep-ph/0309339.

[5] I. Antoniadis, N. A-Hamed, S. Dimopoulos, and G. R. Dvali, New dimensions at a millimeter to a fermi and superstrings at a TeV, Phys.Lett.B436(1998)257 hep-ph/9804398; I. Antoniadis, A possible new dimension at a few TeV, Phys.Lett.B246(1990)37\%; N. A-Hamed, S. Dimopoulos and G. R. Dvali, The hierarchy problem and new dimensions at a millimeter, Phys.Lett.B429(1998)263 hep-ph/9803315.

[6] T. Damour and A. Vilenkin, Gravitational radiation from cosmic (super)strings: bursts, stochastic background, and observational windows hep-th/0410222.

[7] T. Matsuda, Primordial black holes from cosmic necklaces, JHEP 0604:017,2006 hep-ph/0509062; T. Matsuda, Dark matter production from cosmic necklaces, JCAP 0604:005,2006 hep-ph/0509064; T. Matsuda, Primordial black holes from monopoles connected by strings hep-ph/0509061.

[8] T. Matsuda, Weak scale inflation and unstable domain walls, Phys.Lett.B486:300305,2000 hep-ph/0002194, T. Matsuda, On the cosmological domain wall problem in supersymmetric models, Phys.Lett.B436:264-268,1998 hep-ph/9804409.

[9] A. R. Liddle, A. Mazumdar and F. E. Schunck, Assisted inflation, Phys.Rev.D58(1998)061301 astro-ph/9804177, P. Kanti and K. A. Olive, Assisted chaotic inflation in higher dimensional theories, Phys. Lett. B464(1999)192 hep-ph/9906331; N. Arkani-Hamed, S. Dimopoulos, N. Kaloper, and J. MarchRussell, Rapid asymmetric inflation and early cosmology in theories with submillimeter dimensions, Nucl.Phys.B567(2000)189 hep-ph/9903224; R. N. Mohapatra, A. PerezLorenzana, and C. A. de S. Pires, Inflation in models with large extra dimensions driven by a bulk scalar field, Phys.Rev.D62(2000)105030 hep-ph/0003089; A. Mazumdar, S. Panda and A. Perez-Lorenzana, Assisted inflation via tachyon condensation, Nucl.Phys.B614(2001)101 hep-ph/0107058; A. Mazumdar, Extra dimensions and inflation, Phys.Lett.B469(1999)55 hep-ph/9902381; A. M. Green and A. Mazumdar, Dynamics of a large extra dimension inspired hybrid inflation 
model, Phys.Rev.D65(2002)105022 hep-ph/0201209; D. H. Lyth, Inflation with TeV scale gravity needs supersymmetry, Phys.Lett.B448(1999)191 hep-ph/9810320; T. Matsuda, Kaluza-Klein modes in hybrid inflation, Phys.Rev.D66(2002)107301 hep-ph/0209214; T. Matsuda, Successful D term inflation with moduli, Phys.Lett.B423(1998)35 hep-ph/9705448, R. Allahverdi, K. Enqvist, J. GarciaBellido and A. Mazumdar, Gauge invariant MSSM inflaton hep-ph/0605035.

[10] G. R. Dvali, G. Gabadadze, Nonconservation of global charges in the brane universe and baryogenesis, Phys.Lett.B460(1999)47 hep-ph/9904221; A. Masiero, M. Peloso, L. Sorbo, and R. Tabbash, Baryogenesis versus proton stability in theories with extra dimensions, Phys.Rev.D62(2000)063515 hep-ph/0003312; A. Pilaftsis, Leptogenesis in theories with large extra dimensions, Phys.Rev.D60(1999)105023 hep-ph/9906265; R. Allahverdi, K. Enqvist, A. Mazumdar and A. Perez-Lorenzana, Baryogenesis in theories with large extra spatial dimensions, Nucl.Phys. B618(2001)377 hep-ph/0108225; S. Davidson, M. Losada, and A. Riotto, A new perspective on baryogenesis, Phys.Rev.Lett.84(2000)4284 hep-ph/0001301.

[11] T. Matsuda, Baryon number violation, baryogenesis and defects with extra dimensions, Phys.Rev.D66(2002)023508 hep-ph/0204307; T. Matsuda, Activated sphalerons and large extra dimensions, Phys.Rev.D66(2002)047301 hep-ph/0205331; T. Matsuda, Enhanced baryon number violation due to cosmological defects with localized fermions along extra dimensions, Phys.Rev.D65(2002)107302 hep-ph/0202258; T. Matsuda, Defect mediated electroweak baryogenesis and hierarchy, J.Phys.G27(2001)L103 hep-ph/0102040.

[12] T. Matsuda, Hybridized Affleck-Dine baryogenesis, Phys.Rev.D67(2003)127302 hep-ph/0303132; T. Matsuda, Affleck-Dine baryogenesis after thermal brane inflation, Phys.Rev.D65(2002)103501 hep-ph/0202209; T. Matsuda, Affleck-Dine baryogenesis in the local domain, Phys.Rev.D65(2002)103502 hep-ph/0202211; T. Matsuda, Electroweak baryogenesis mediated by locally supersymmetry breaking defects, Phys.Rev.D64(2001)083512 hep-ph/0107314. 
[13] S. Mollerach, Isocurvature baryon perturbations and inflation, Phys.Rev.D42(1990)313; A. D. Linde, V. Mukhanov, Nongaussian Isocurvature Perturbations from Inflation, Phys.Rev.D56(1997)535 astro-ph/9610219; K. Enqvist, M. S. Sloth, Adiabatic CMB perturbations in pre-big bang string cosmology, Nucl.Phys.B626(2002)395 hep-ph/0109214.

[14] T. Moroi, T. Takahashi, Effects of Cosmological Moduli Fields on Cosmic Microwave Background, Phys.Lett.B522(2001)215 hep-ph/0110096 D. H. Lyth, D. Wands, Generating the curvature perturbation without an inflaton, Phys.Lett.B524(2002)5 hep-ph/0110002.

[15] K. Dimopoulos, G. Lazarides, D. Lyth and R. R. de Austri, Curvaton dynamics,Phys.Rev.D68:123515,2003 hep-ph/0308015.

[16] K. Dimopoulos, D. H. Lyth, Models of inflation liberated by the curvaton hypothesis, Phys.Rev.D69(2004)123509,2004 hep-ph/0209180; M. Giovannini, Tracking curvaton(s)?, Phys.Rev.D67(2003)123512 hep-ph/0310024; T. Matsuda, Curvaton paradigm can accommodate multiple low inflation scales, Class.Quant.Grav.21(2004)L hep-ph/0312058.

[17] T. Matsuda, Topological curvatons, Phys.Rev.D72:123508,2005 hep-ph/0509063, T. Matsuda, to appear.

[18] T. Matsuda, Brane necklaces and brane coils, JHEP 0505(2005)015 hep-ph/0412290.

[19] T. Matsuda, Brane $Q$ Ball, branonium and brane $Q$ ball inflation, JCAP 0410:014,2004 hep-ph/0402223.

[20] T.Matsuda, Formation of cosmological brane defects, JHEP 0411:039,2004 hep-ph/0402232; T. Matsuda, Formation of monopoles and domain walls after brane inflation, JHEP 0410(2004)042 hep-ph/0406064.

[21] T. Matsuda, Incidental Brane Defects, JHEP 0309(2003)064 hep-th/0309266. 
[22] T. Matsuda, String production after angled brane inflation, Phys.Rev.D70(2004)023502 hep-ph/0403092.

[23] M. Eto, T. Fujimori, Y. Isozumi, M. Nitta, K. Ohashi, K. Ohta and N. Sakai, it NonAbelian Vortices on Cylinder - Duality between vortices and walls hep-th/0601181.

[24] N. Jones, H. Stoica, and S. H. H. Tye, Brane interaction as the origin of inflation, JHEP 0207(2002)051 hep-th/0203163; S. Sarangi, S. H. H. Tye, Cosmic string production towards the end of brane inflation, Phys.Lett.B536(2002)185 hep-th/0204074; L. Pogosian, S. H. H. Tye, I. Wasserman and M. Wyman, Observational constraints on cosmic string production during brane inflation, Phys.Rev.D68(2003)023506 hep-th/0304188.

[25] T. Matsuda, PBH and DM from cosmic necklaces, Talk given at 15th Workshop on General Relativity and Gravitation (JGRG 15), Tokyo, Japan, 28 Nov - 2 Dec 2005 hep-ph/0601014.

[26] S. Kachru, R. Kallosh, A. Linde, J. M. Maldacena, L. McAllister, and S. P. Trivedi, Towards inflation in string theory, JCAP 0310:013,2003 hep-th/0308055

[27] M. Alishahiha, E. Silverstein,and D. Tong, DBI in the sky, Phys.Rev.D70:123505,2004 hep-th/0404084; E. Silverstein and D. Tong, Scalar speed limits and cosmology: Acceleration from D-cceleration, Phys.Rev.D70:103505,2004 hep-th/0310221.

[28] H. Singh, (A)symmetric tachyon rolling in de Sitter spacetime: An Universe devoid of planck density, Nucl.Phys.B734:169-184,2006 hep-th/0508101.

[29] K. Dimopoulos and M. Axenides, Hybrid inflation without flat directions and without primordial black holes, JCAP 0506:008,2005 hep-ph/0310194.

[30] A. Linde, Fast roll inflation, JHEP 0111:052,2001 hep-th/0110195.

[31] G. Dvali and S. Kachru, New old inflation, From fields to strings, vol. 2* 1131 hep-th/0309095 
[32] A. A. Starobinsky, Multicomponent De Sitter (Inflationary) Stages And The Generation Of Perturbations, JETP Lett.42:152-155,1985, Pisma Zh.Eksp.Teor.Fiz.42:124$127,1985$.

[33] D. H. Lyth, Generating the curvature perturbation at the end of inflation, JCAP 0511:006,2005 astro-ph/0510443, D. H. Lyth and Y. Rodriguez, Inflationary Prediction for Primordial Non-Gaussianity astro-ph/0504045, L. Boubekeur and D. H. Lyth, Detecting a small perturbation through its non-Gaussianity, Phys.Rev. D73 (2006) 021301 astro-ph/0504046,

[34] M. Sasaki and E. D. Stewart, A General analytic formula for the spectral index of the density perturbations produced during inflation, Prog.Theor.Phys.95:71-78,1996 astro-ph/9507001, D. H. Lyth, K. A. Malik and M. Sasaki, it A General proof of the conservation of the curvature perturbation, JCAP 0505:004,2005 astro-ph/0411220, M. Sasaki and T. Tanaka Superhorizon scale dynamics of multiscalar inflation, Prog. Theor.Phys.99:763-782,1998 gr-qc/9801017.

[35] S. Kachru, R. Kallosh, A. Linde and S. P. Trivedi, De Sitter vacua in string theory, Phys.Rev.D68:046005,2003 hep-th/0301240.

[36] O. Aharony, Y. E. Antebi and M. Berkooz, Open string moduli in KKLT compactifications, Phys.Rev.D72:106009,2005 hep-th/0508080 